\title{
Adoption of Multi-society Guidelines Facilitates Value-Based Reduction in Screening and Surveillance Colonoscopy Volume During COVID-19 Pandemic
}

\author{
Alexander Hua Xiao ${ }^{1}$. Stephen Y. Chang ${ }^{1,2} \cdot$ Christian G. Stevoff $^{1,2} \cdot$ Srinadh Komanduri $^{1,2} \cdot$ John E. Pandolfino $^{1,2}$. \\ Rajesh N. Keswani ${ }^{1,2}$ (D)
}

Received: 5 June 2020 / Accepted: 5 August 2020 / Published online: 16 August 2020

(c) Springer Science+Business Media, LLC, part of Springer Nature 2020

\begin{abstract}
Background COVID-19 has caused a backlog of endoscopic procedures; colonoscopy must now be prioritized to those who would benefit most. We determined the proportion of screening and surveillance colonoscopies appropriate for rescheduling to a future year through strict adoption of US Multi-Society Task Force (USMSTF) guidelines.

Methods We conducted a single-center observational study of patients scheduled for "open-access colonoscopy"- - ordered by a primary care provider without being seen in gastroenterology clinic-over a 6-week period during the COVID-19 pandemic. Each chart was reviewed to appropriately assign a surveillance year per USMSTF guidelines including demographics, colonoscopy history and family history. When guidelines recommended a range of colonoscopy intervals, both a "conservative" and "liberal" guideline adherence were assessed.

Results We delayed 769 "open-access" screening or surveillance colonoscopies due to COVID-19. Between 14.8\% (conservative) and $20.7 \%$ (liberal), colonoscopies were appropriate for rescheduling to a future year. Conversely, 415 (54.0\%) patients were overdue for colonoscopy. Family history of CRC was associated with being scheduled too early for both screening (OR 3.9; CI 1.9-8.2) and surveillance colonoscopy (OR 2.6, CI 1.0-6.5). The most common reasons a colonoscopy was inappropriately scheduled this year were failure to use new surveillance colonoscopy intervals (28.9\%), incorrectly applied family history guidelines (27.2\%) and recommending early surveillance colonoscopy after recent normal colonoscopy (19.3\%).

Conclusion Up to one-fifth of patients scheduled for "open-access" colonoscopy can be rescheduled into a future year based on USMSTF guidelines. Rigorously applying guidelines could judiciously allocate colonoscopy resources as we recover from the COVID-19 pandemic.
\end{abstract}

Keywords Colonoscopy overuse $\cdot$ COVID- $19 \cdot$ Surveillance colonoscopy

$\begin{array}{ll}\text { Abbreviations } \\ \text { USMSTF } & \text { United States Multi-Society Task Force } \\ \text { EHR } & \text { Electronic health record } \\ \text { OR } & \text { Odds ratio } \\ \text { CI } & 95 \% \text { Confidence interval }\end{array}$

Electronic supplementary material The online version of this article (https://doi.org/10.1007/s10620-020-06539-1) contains supplementary material, which is available to authorized users.

Rajesh N. Keswani

raj-keswani@northwestern.edu

1 Northwestern University Feinberg School of Medicine, Chicago, IL, USA

2 Digestive Health Center, Northwestern Medicine, 676 N. St. Clair, Suite 1400, Chicago, IL 60611, USA

\section{Introduction}

The introduction of the novel coronavirus (caused by SARSCoV-2) has disrupted all aspects of the US healthcare system. Due to a need to conserve vital healthcare resources including personal protective equipment, protect healthcare personnel and maintain social distancing, gastroenterology societies recommended the delay of elective procedures at the onset of the pandemic. In gastroenterology, nearly all practices adopted this policy which resulted in a rapid and dramatic reduction in gastrointestinal endoscopy clinical volume $[1,2]$. Similarly, most practices now anticipate a very slow reintroduction of clinical volume as restrictions are lifted.

This reduction in endoscopy volume during the months of the pandemic will invariably result in a large backlog 
of procedures. Although numerous operational adjustments can be considered to distribute procedure volume-including weekend days and evening hours-it is unclear whether these will be enough to sufficiently handle volume. Thus, given the limited resource of endoscopy teams, it is critically important to ensure that patients undergoing endoscopy are appropriately triaged and rescheduled to the appropriate year. In 2017, the US Multi-Society Task Force (USMSTF) published guidelines [3], updated from 2008 [4], on appropriate use of screening colonoscopy; more recently in 2020, updated guidelines [5], previously published in 2012 [6], were published regarding appropriate use of surveillance colonoscopy. In general, these updated guidelines recognize a decreased need for surveillance colonoscopy at short intervals.

We hypothesized that adoption of new guidelines would reduce the volume of colonoscopy procedures scheduled for this calendar year, shifting the limited available resources due to the COVID-19 pandemic to those patients who most benefit from colonoscopy this year. Thus, the primary aim of this study was to determine what proportion of open-access colonoscopies could appropriately be rescheduled in a new calendar year with strict adoption of USMSTF colonoscopy guidelines.

\section{Methods}

\section{Study Design and Setting}

We conducted an observational study of patients scheduled for "open-access colonoscopy" at a single urban academic medical center over a six-week period (March 16, 2020-May 1, 2020). Our Institution's Review Board approved the study (institutional review board STU00212570). A waiver of informed consent was obtained.

\section{Inclusion and Exclusion Criteria}

All patients aged 18-89 who were scheduled for "openaccess" colonoscopy over the 6-week period were included in this analysis. An open-access colonoscopy signifies a colonoscopy directly ordered by the primary care provider that occurs without the patient first seeing a gastroenterology provider in clinic and represents the predominant means by which colonoscopies are performed at our institution. These patients are scheduled based on the recommendation of the primary care provider and are screened for appropriateness using a standardized checklist. At the scheduling process, patients who report that the colonoscopy is being requested primarily for new symptoms are generally referred for a clinic visit. Both screening and surveillance colonoscopies can be scheduled directly via a primary care provider without requiring the patient to be seen by a gastroenterology provider. Patients who had seen a gastroenterology physician or advanced practice provider in the past for an alternate complaint (e.g., reflux) were included.

Patients referred for a diagnostic colonoscopy (work-up of a positive stool test or symptoms) were excluded from this analysis.

\section{Intervention}

At the onset of the COVID-19 pandemic, all elective gastrointestinal endoscopy procedures at our institution were delayed. We anticipated that adopting this policy would most significantly affect patients with existing appointments for predominantly screening and surveillance colonoscopy.

Each patient chart was reviewed for all relevant information required to appropriately assign a surveillance year per existing USMSTF guidelines (Supplemental Table). Patient data included age, race, gender, prior history of colorectal cancer (CRC), as well as relevant family history (number and age of relatives diagnosed with CRC or advanced adenomas/serrated polyps larger than $10 \mathrm{~mm}$ ). When patients reported a family history of a first degree relative with CRC at an undocumented age, they were treated as if the age of diagnosis for the family member was $<60$. The indication for scheduled colonoscopy was recorded, as was any history of prior gastroenterology visits. For patients who had undergone prior colonoscopy, we also abstracted data from the two previous colonoscopies for procedure dates, indications, adequacy of preparation, completion of the colonoscopy, as well as number, type and size of polyps detected during those colonoscopies.

These data were used to determine the appropriate surveillance year for each patient based on USMSTF guidelines $[3,5]$ (Supplemental Table).

When surveillance guidelines recommended a range of surveillance choices, both a "conservative" and "liberal" adherence to guidelines were assessed. For example, after removal of a single adenoma in average risk patients, current surveillance guidelines recommend a repeat colonoscopy in 7-10 years: in the conservative approach, patients currently scheduled within 6 years (i.e., before the shortest end of guideline recommendations) of colonoscopy are appropriate for rescheduling to a future year; in the liberal approach, patients scheduled within 9 years (i.e., before the longest end of guideline recommendations) are appropriate for rescheduling. Similarly, a conservative and liberal interpretation of screening guidelines was utilized. In the liberal approach, non-African American patients scheduled for screening colonoscopy before the ages 45-49 were considered appropriate for rescheduling to a future year; in the conservative approach, all screening beginning at age 45 (regardless of race) were considered appropriate for this calendar year 
based on prior American Cancer Society guidance [7]. All patients considered for rescheduling in a future year were manually reviewed by one of the three attending gastroenterologists (SC, CS, RNK).

\section{Study Outcomes}

The primary study outcome was the percentage of colonoscopies scheduled this calendar year that were not adherent to current USMSTF guidelines for screening [3] and surveillance [5] colonoscopy (Supplemental Table).

\section{Statistical Analysis}

A complete case analysis was performed, we did not anticipate any missing data, and all analyses were planned a priori. $p$ values $<0.05$ were considered statistically significant. Values were reported as proportions. Categorical variables were compared using Chi-squared test, and continuous variables were compared using the Student's $t$ test where appropriate.

\section{Results}

\section{Patient Population}

Of the 791 patients, 22 (2.7\%) patients were referred for a diagnostic colonoscopy including 9 patients with a recent positive stool-based screening test. These 22 patients were excluded from further analysis. We identified 769 patients

Table 1 Procedure indications $(n=791)$

\begin{tabular}{ll}
\hline First screening colonoscopy & $331(41.8 \%)$ \\
Follow-up screening colonoscopy & $221(27.9 \%)$ \\
Surveillance colonoscopy & $217(27.4 \%)$ \\
Diagnostic colonoscopy & $22(2.8 \%)$ \\
\hline
\end{tabular}

scheduled for screening or surveillance colonoscopy (mean age 58.9, 53.6\% female) with one of 18 colonoscopists; 552 $(69.7 \%)$ patients were scheduled for screening colonoscopy and 217 (27.4\%) patients were scheduled for surveillance colonoscopy (Table 1). Overall, 97 patients (12.2\%) reported a history of a first degree relative with CRC or prior advanced adenoma including 70 patients with a first degree relative with CRC at an age $<60(n=39)$ and/or unknown age $(n=33)$. Nine patients $(1.1 \%)$ had a personal history of CRC. Fourteen patients (1.8\%) were due for a repeat colonoscopy for a previously incomplete examination or inadequate bowel preparation.

\section{Overall Adherence to USMSTF Guidelines}

Among the 769 patients scheduled for screening or surveillance colonoscopy, 159 (20.7\%) patients met criteria to be rescheduled in a new calendar year using a liberal interpretation of screening and surveillance guidelines. Using a conservative interpretation of guidelines, 114 (14.8\%) patients were appropriate for rescheduling in a future year (Table 2). In contrast, 415 (54.0\%) patients were overdue for screening/surveillance colonoscopy including 103 patients (13.4\%) who were overdue at least 5 years. Of the 103 patients overdue for colonoscopy $\geq 5$ years, nearly all $(93.2 \%)$ were scheduled for an initial or follow-up screening colonoscopy.

\section{Screening Colonoscopy Adherence to USMSTF Guidelines}

Of the subgroup of 552 patients scheduled for screening colonoscopy, 331 were scheduled for their first screening colonoscopy and 221 patients were scheduled for follow-up screening colonoscopy (i.e., repeat screening colonoscopy after a prior negative examination). Of the 331 patients scheduled for first-time screening colonoscopy, $4(1.2 \%)$ were scheduled "early" using conservative guidelines-in other words, these patients were scheduled

Table 2 Non-adherence to screening and surveillance colonoscopy guidelines $(n=769)$

Overall non-adherence using conservative interpretation of guidelines

$114(14.8 \%)$

Overall non-adherence using liberal interpretation of guidelines

$159(20.7 \%)$

Overdue for screening or surveillance colonoscopy

$415(54.0 \%)$

First screening colonoscopy $(n=331)$

Non-adherence using conservative guidelines (scheduled at age $<45$ without significant family history)

$4(1.2 \%)$

Non-adherence using liberal guidelines (scheduled age $<50[$ AA $<45]$ without significant family history)

Follow-up screening colonoscopy $(n=221)$

Non-adherence $(<10$ years after last normal colonoscopy without FDR; $<5$ years with single FDR with CRC or advanced adenoma)

Surveillance colonoscopy $(n=217)$

Non-adherence using conservative guidelines 
before the age of 45 without a first degree relative with CRC or advanced neoplasia. Using a liberal interpretation of guidelines (patients scheduled for first colonoscopy before the age of 50 [or 45 for African Americans]), 35 patients $(10.6 \%)$ were appropriate for rescheduling to a new calendar year (Table 2).

Among the 221 patients scheduled for follow-up screening colonoscopy, $39(17.6 \%)$ were scheduled at least 1 year early (mean 3.6 years early) by liberal interpretation of guidelines. Among patients scheduled for a follow-up screening colonoscopy, only a family history of a first degree relative with CRC was associated with risk of being scheduled too early for colonoscopy (Odds Ratio [OR] 3.9; 95\% Confidence Interval [CI] 1.9-8.2). Patient age, sex, race and a distant family history of CRC were not associated with being scheduled for a repeat screening colonoscopy at an inappropriately early interval.

\section{Surveillance Colonoscopy Adherence to USMSTF Guidelines}

Among the 217 patients scheduled for surveillance colonoscopy, 71 (32.7\%) were appropriate for rescheduling in a future year using a conservative interpretation of guidelines (i.e., use of the lower range of surveillance intervals per guidelines). Using liberal application of guidelines (i.e., using the upper range of surveillance intervals per guidelines), 85 patients (39.2\%) were appropriate for scheduling in a new calendar year. Older patient age (65.9 years vs. 63.2 years, $p=0.02$ ) and a family history of a first degree relative with CRC (OR 2.6, CI 1.0-6.5) were associated with being scheduled for surveillance colonoscopy inappropriately early. Patient race, gender and a family history of a second degree relative with CRC were not associated with non-adherence to surveillance colonoscopy guidelines.

\section{Root Cause Analysis for Etiologies of Non-adherence}

We classified the suspected causes of non-adherence in the 114 patients scheduled early using a conservative interpretation of the guidelines, 43 of whom were screening patients and 71 of whom were scheduled for early surveillance (Table 3). Approximately one-quarter of colonoscopies scheduled inappropriately early were due to incorrectly applied family history guidelines (e.g., screening colonoscopy after 5 years due to a history of a first degree relative at an age $>60$ ). Among the 71 surveillance colonoscopy patients, 11 patients were inappropriately scheduled due to misapplication of both recent guidelines and older guidelines (e.g., average risk patient told to return 3 years after removal of diminutive adenoma). Approximately one-third of surveillance colonoscopies were scheduled inappropriately early due to a distant history of colon neoplasia but with one or more normal recent colonoscopies. Finally, nearly half of the inappropriately scheduled surveillance colonoscopies were appropriate for rescheduling in a future calendar year based only per the most recent USMSTF guidelines (e.g., told to return at 5 years after removal of a diminutive adenoma; recent guidelines recommend a 7-10-year surveillance interval).

\section{Incomplete Medical Records' Effect on Guideline Adherence}

We identified 71 patients $(8.9 \%)$ who self-reported the date of their most recent colonoscopy but where there was no colonoscopy report available to the referring primary care provider. Of these, $20(2.5 \%)$ patients appeared to be scheduled early for their screening $(n=15)$ or surveillance $(n=5)$ colonoscopy; we were unable to confirm these for rescheduling due to these incomplete/unavailable records. Similarly, 33 patients $(4.3 \%)$ reported a first degree relative with CRC without a listed age, $11(1.3 \%)$ of whom might be

Table 3 Root causes of inappropriate early screening and surveillance colonoscopy

\begin{tabular}{|c|c|c|c|}
\hline & Screening $(n=43)$ & Surveillance $(n=71)$ & Overall $(n=114)$ \\
\hline \multicolumn{4}{|l|}{ Causes of inappropriate screening or surveillance colonoscopy } \\
\hline Incorrectly applied family history guidelines & $27(62.8 \%)$ & $4(5.6 \%)$ & $31(27.2 \%)$ \\
\hline Recent colonoscopy with adequate but not ideal preparation & $2(4.7 \%)$ & $1(1.4 \%)$ & $3(2.6 \%)$ \\
\hline Early screening without reason & $12(27.9 \%)$ & N/A & $12(10.5 \%)$ \\
\hline Surveillance after removal of a non-neoplastic polyp & $2(4.7 \%)$ & $0(0 \%)$ & $2(1.8 \%)$ \\
\hline \multicolumn{4}{|l|}{ Causes of inappropriate surveillance colonoscopy } \\
\hline Early surveillance with last colonoscopy normal & N/A & $22(31.0 \%)$ & $22(19.3 \%)$ \\
\hline $\begin{array}{l}\text { Early surveillance (recent colonoscopy with neoplasia) based upon } \\
\text { old (2012) and recent (2020) guidelines }\end{array}$ & N/A & $11(15.5 \%)$ & $11(9.6 \%)$ \\
\hline $\begin{array}{l}\text { Early surveillance (recent colonoscopy with neoplasia) based upon } \\
\text { recent (2020) guidelines only }\end{array}$ & N/A & $33(46.5 \%)$ & $33(28.9 \%)$ \\
\hline
\end{tabular}


rescheduled if an accurate age of CRC could be ascertained. In total, an additional 31 patients (4.0\%) might be appropriate for rescheduling in a future year if we had colonoscopy records available.

When excluding patients without prior colonoscopy reports for review, we found that the follow-up screening colonoscopy non-adherence rate was $22.9 \%$ and surveillance colonoscopy non-adherence rate was $39.4 \%$ using a liberal interpretation of the guidelines. Similarly, using a conservative interpretation of the guidelines, the follow-up screening colonoscopy non-adherence rate was $22.2 \%$ and surveillance colonoscopy non-adherence rate was $32.7 \%$.

\section{Discussion}

As endoscopy units recover from the COVID-19 pandemic, it is critical to focus the limited endoscopy resources upon those patients most in need of colonoscopy this year. We hypothesized that a significant proportion of our open colonoscopy patient panel - those patients that are scheduled for a colonoscopy without seeing a gastroenterology providercould be safely rescheduled to a future year based on adhering to USMSTF colonoscopy guidelines. Our results suggest that following existing guidelines would shift $15-21 \%$ of "open-access" patients at our institution to a future, appropriate year based on either a conservative or liberal interpretation of existing guidelines. Interestingly, just over half of the patients were overdue for screening or surveillance colonoscopy, highlighting the importance of shifting valuable resources to those patients most in need of colonoscopy.

We identified several factors contributing to patients being scheduled for a colonoscopy incorrectly this year. Of the patients scheduled inappropriately for a colonoscopy this year, nearly half were non-adherent to the most recent USMSTF surveillance colonoscopy guidelines. In general, these recent surveillance guidelines de-emphasize surveillance colonoscopy intervals. For example, colonoscopy is now recommended 7-10 years after removal of a single adenoma $<10 \mathrm{~mm}$, though we found that most patients are still being scheduled at 5-year intervals. We also identified a significant proportion of patients who were non-adherent to any recent colonoscopy surveillance guidelines (e.g., surveillance colonoscopy in 5 years due to a distant history of adenomas). Our results suggest that strict adherence to colonoscopy surveillance guidelines would reduce the burden of surveillance of colonoscopy by 33-39\%, potentially freeing limited endoscopy space for patients who are at most need for colonoscopy. Previous studies have similarly identified that both endoscopists and primary care physicians tend to recommend repeat colonoscopies sooner than guidelines [8-10]. Within the Veterans Affair's health system, non-adherence with guidelines averaged $36 \%$, with greater non-adherence (45-52\%) for colonoscopies following polyp removal. Presumably, fear of missed cancer would drive overuse of endoscopy as well as lack of awareness or disagreement with guidelines; these factors were found to be associated with non-adherence [11]. Other factors associated with non-adherence included bowel preparation quality, geographic region, Charlson comorbidity score and colonoscopy indication [9].

Our data also suggest a lack of understanding around appropriate use of family history to guide screening and surveillance colonoscopy. Inaccurate application of family history guidelines accounted for approximately one-quarter of all patients who were scheduled too early. Specifically, we found that providers often recommend early colonoscopy intervals for patients with lower-risk family history (e.g., first degree relative with colorectal cancer after the age of 60 or second degree relative with colorectal cancer). Finally, we found multiple issues related to inadequate documentation and fragmented healthcare records leading to early colonoscopy. We estimated that approximately $4 \%$ of patients could be rescheduled for a future colonoscopy year if there were better details available regarding prior colonoscopies or accurate documentation of family history.

There is increasing concern regarding the backlog of endoscopy procedures and the impact that the delay related to COVID-19 will have upon patient health. While providers anticipate adding weekday and weekend hours [1], this limited resource should be utilized on those patients most in need [12]. Prior work suggests that cessation of services may result in a significant increase in advanced malignancies such as gastrointestinal disease, due the backlog of procedures delaying necessary screening and surveillance compounded by patients' dismissing symptoms (including change in bowel habits, fatigue and weight loss) as "trivial" during the ongoing COVID-19 pandemic [13]. Our data emphasize that practices must not look at the backlog of patients as a whole but should individually target those patients most at risk for CRC first. While one-quarter of our patients could safely be rescheduled in another year, just over half of the patients are overdue for colonoscopy. These patients should be brought in for CRC screening prior to considering those patients who were scheduled early. Furthermore, as a significant proportion of patients may be unwilling to schedule a needed colonoscopy during the pandemic, resources to encourage colonoscopy adherence should only be allocated to those patients who are actually due for colonoscopy based on the newest guidelines [14].

In aggregate, our data suggest a need to simplify the process by which colonoscopy screening and surveillance intervals are calculated. We propose several methods which can be utilized to both reduce errors and adjust intervals based upon new guidelines. The electronic health record (EHR) should be optimized to incorporate guidelines at two 
points of care: following the colonoscopy and in the primary care provider office. This approach should incorporate data that already exists within the EHR and thus not rely on provider input. Areas where the data are incomplete, such as the age of CRC in a relative, should result in a prompt to clinicians to correct these missing data. These accurate data will facilitate easier and more precise calculation of colonoscopy intervals. Some institutions have introduced this sort of EHR-embedded clinical decision tool, such as The Parkland-UT Southwestern Colonoscopy Reporting System (CoRS), and have found that in a majority of cases it improved adherence to guideline-based surveillance recommendations while also being easy to use and reducing the physician's workload $[15,16]$. More recently, an alternative approach using natural language processing to automatically calculate colonoscopy intervals has been described, but this requires further validation in additional centers [17].

However, it is important to understand that many patients were incorrectly scheduled based on the specific recommendation of the colonoscopist, suggesting either a lack of awareness or disagreement with existing colonoscopy guidelines. Previous work has similarly identified a lack of awareness or desire to follow surveillance guidelines. Though gastroenterologists reported being "aware" of USMSTF guidelines, they often recommended colonoscopy intervals that adhered poorly to guidelines when presented with clinical vignettes [18]. Earlier work has suggested that this non-adherence is related, in part, to discomfort with reducing colonoscopy surveillance intervals [19] even in managed care settings [9]. This lack of provider alignment with colonoscopy guidelines will require further education which would ideally be delivered at the time providers are writing procedure reports and reconciling pathology results.

There are several strengths to our study. Notably, this is the largest study to determine the impact of applying the most recent USMSTF guidelines to colonoscopy volumes. Furthermore, this represents the practice of a large group of screening/surveillance colonoscopists. However, we note several important limitations of our study. We report the findings of a single academic medical center, and thus, our results may not be generalizable to community practices. Whether colonoscopy overuse is more or less prevalent in other settings requires study. We also are unable to assess what proportion of patients is willing to now be rescheduled to a future year with more strict application of screening/ surveillance guidelines.

In summary, we found that up to one-fifth of patients scheduled for an "open-access" colonoscopy during a sixweek interval through the COVID-19 pandemic could appropriately be rescheduled into a new calendar year whereas one-half of patients were overdue for colonoscopy. The most common reason for patients being scheduled early was due to inappropriate application of colonoscopy intervals related to prior history of colon polyps and family history of colorectal cancer. Our findings support the need to implement guidelines at the time of colonoscopy as well as scheduling. We propose that centers can more rigorously apply guideline-based care to reduce the backlog of colonoscopy procedures accrued during the COVID-19 pandemic and focus resources on those patients in most need of colonoscopy.

Author's contribution Conception: RNK, SYC, CS, JEP; Design: AHX, RNK; Data Acquisition: AHX, SYC, CS, RNK; Analysis and interpretation of the data: AHX, RNK; Drafting of the article: AHX, RNK; Critical revision of the article for important intellectual content: AHX, SYC, CS, SK, JEP, RNK; Final approval of the article: AHX, SYC, CS, SK, JEP, RNK.

Funding None.

\section{Compliance with Ethical Standards}

Conflict of interest The authors declare that they have no conflict of interest.

\section{References}

1. Forbes N, Smith ZL, Spitzer RL, Keswani RN, Wani SB, Elmunzer BJ. Changes in gastroenterology and endoscopy practices in response to the COVID-19 pandemic: results from a North American survey. Gastroenterology. 2020. https://doi. org/10.1053/j.gastro.2020.04.071.

2. Repici A, Pace F, Gabbiadini R, et al. Endoscopy units and the COVID-19 outbreak: A multi-center experience from Italy. Gastroenterology. 2020;159:363-366.

3. Rex DK, Boland CR, Dominitz JA, et al. Colorectal cancer screening: recommendations for physicians and patients from the U.S. multi-society task force on colorectal cancer. Gastroenterology. 2017;153:307-323.

4. Levin B, Lieberman DA, McFarland B, et al. Screening and surveillance for the early detection of colorectal cancer and adenomatous polyps, 2008: a joint guideline from the American Cancer Society, the US Multi-Society Task Force on Colorectal Cancer, and the American College of Radiology. CA Cancer J Clin. 2008;58:130-160.

5. Gupta S, Lieberman D, Anderson JC, et al. Recommendations for follow-up after colonoscopy and polypectomy: a consensus update by the US multi-society task force on colorectal cancer. Gastroenterology. 2020;158:1131e1135-1153e1135.

6. Lieberman DA, Rex DK, Winawer SJ, et al. Guidelines for colonoscopy surveillance after screening and polypectomy: a consensus update by the US Multi-Society Task Force on Colorectal Cancer. Gastroenterology. 2012;143:844-857.

7. Wolf AMD, Fontham ETH, Church TR, et al. Colorectal cancer screening for average-risk adults: 2018 guideline update from the American Cancer Society. CA Cancer J Clin. 2018;68:250-281.

8. Boolchand V, Olds G, Singh J, Singh P, Chak A, Cooper GS. Colorectal screening after polypectomy: a national survey study of primary care physicians. Ann Internal Med. 2006;145:654-659.

9. Johnson MR, Grubber J, Grambow SC, et al. Physician non-adherence to colonoscopy interval guidelines in the veterans affairs healthcare system. Gastroenterology. 2015;149:938-951. 
10. Mysliwiec PA, Brown ML, Klabunde CN, Ransohoff DF. Are physicians doing too much colonoscopy? A national survey of colorectal surveillance after polypectomy. Ann Internal Med. 2004;141:264-271.

11. Patel N, Tong L, Ahn C, Singal AG, Gupta S. Post-polypectomy guideline adherence: importance of belief in guidelines, not guideline knowledge or fear of missed cancer. Dig Dis Sci. 2015;60:2937-2945.

12. Sultan S, Lim JK, Altayar O, et al. AGA Institute rapid recommendations for gastrointestinal procedures during the COVID-19 pandemic. Gastroenterology. 2020. https://doi.org/10.1053/j.gastr o.2020.03.072.

13. Jones D, Neal RD, Duffy SRG, Scott SE, Whitaker KL, Brain K. Impact of the COVID-19 pandemic on the symptomatic diagnosis of cancer: the view from primary care. Lancet Oncol. 2020;21:748-750.

14. Armellini E, Repici A, Alvisi C, et al. Analysis of patients attitude to undergo urgent endoscopic procedures during COVID-19 outbreak in Italy. Dig Liver Dis. 2020;52:695-699.

15. Magrath M, Yang E, Ahn C, et al. Impact of a clinical decision support system on guideline adherence of surveillance recommendations for colonoscopy after polypectomy. J Natl Compr Cancer Netw. 2018;16:1321-1328.
16. Skinner CS, Gupta S, Halm EA, et al. Development of the Parkland-UT Southwestern Colonoscopy Reporting System (CoRS) for evidence-based colon cancer surveillance recommendations. J Am Med Inform Assoc. 2016;23:402-406.

17. Karwa A, Patell R, Parthasarathy G, Lopez R, McMichael J, Burke CA. Development of an automated algorithm to generate guideline-based recommendations for follow-up colonoscopy Clin Gastroenterol Hepatol. 2020;18:2038-2045.

18. Iskandar H, Yan Y, Elwing J, Early D, Colditz GA, Wang JS. Predictors of poor adherence of US gastroenterologists with colonoscopy screening and surveillance guidelines. Dig Dis Sci. 2015;60:971-978.

19. Saini SD, Nayak RS, Kuhn L, Schoenfeld P. Why don't gastroenterologists follow colon polyp surveillance guidelines? Results of a national survey. J Clin Gastroenterol. 2009;43:554-558.

Publisher's Note Springer Nature remains neutral with regard to jurisdictional claims in published maps and institutional affiliations. 